\title{
CARRIER DEPLETION AND GRAIN MISORIENTATIONS ON INDIVIDUAL GRAIN BOUNDARIES OF POLYCRYSTALLINE SI THIN FILMS
}

\author{
C.-S. Jiang, H.R. Moutinho, F. Liu, M.J. Romero, and M.M. Al-Jassim \\ National Renewable Energy Laboratory (NREL), 1617 Cole Blvd., Golden, CO 80401
}

\begin{abstract}
Structural and microelectrical properties of grain boundaries (GBs) in polycrystalline $\mathrm{Si}$ thin films were investigated by electron backscattering diffraction (EBSD) and scanning capacitance microscopy (SCM). The SCM measurements revealed highly nonuniform carrier depletions among the GBs, indicating the variety of electrical properties due to the specific GB structures. The EBSD measurement showed that the films are weakly [001]-oriented with small fractions of grains in the [111] and [110] orientations. Comparison of the SCM and EBSD measurements taken on the same film area led to the following observations: (1) $\Sigma 3$ GBs do not exhibit carrier depletions and thus do not have charged deep levels; (2) Some $\Sigma 9$ GBs exhibit carrier depletions and some do not, indicating that the intrinsic $\Sigma 9$ GBs do not have charged deep levels and the carrier depletions are due to impurity gettering at the GBs; (3) No significant relationship between the carrier depletion behavior and the grain misorientation was found so far on the GBs with random misorientations; (4) The carrier depletion behavior does not depend only on the grain misorientation but also on the facet where the GB is taken.
\end{abstract}

\section{INTRODUCTION}

Grain boundaries (GBs) in polycrystalline $\mathrm{Si}$ thin films could play a critical role in determining the electronic properties of electronic devices such as solar cells and thin-film transistors [1,2]. In solar cell applications $[3,4]$, most literature $[1,4-8]$ reports that GBs are one of the main causes for device degradation, because the GBs possess deep levels that are harmful recombination centers for photo-excited minority carriers. Moreover, carrier transport over the $p-n$ junction via recombination at the GB defect states increases dark saturate current. On the other hand, a positive role of the GBs was proposed by a two-dimensional device simulation and by a quantum efficiency analysis, suggesting that the GBs help to collect minority carriers if the GBs are doped to a considerable length scale by impurities in the emitter $[9,10]$.

The structure and electronic properties of GBs in polycrystalline $\mathrm{Si}$ films have been studied over several decades [11-25]. However, most measurement and characterization studies on the electronic properties of thin films were carried out on a large section of a sample that contained a huge amount of microscopic GBs, resulting in the characterization of the collective effects of these GBs. On the other hand, the microelectronic properties on individual GBs are expected to depend on a specific GB structure, which varies markedly among GBs [10-16]. Therefore, it is necessary to measure and characterize the microelectronic properties of individual GBs. Both structural and electronic measurements on the same individual GBs are expected to elucidate the GB physics and their effects on the device performance [1416].

In this paper, we report our recent characterization results toward the understanding of the relationship between the structural and microelectronic properties of individual GBs. We first measured carrier depletions on individual GBs using scanning capacitance microscopy (SCM), and resolved various carrier depletion behaviors among the GBs. We then measured the grain misorientations adjacent to the GBs using electron backscattering diffraction (EBSD). These grain misorientations provided information on the GB structures. Finally, we performed SCM and EBSD measurements on the same GBs, and tried to correlate the grain misorientation and carrier depletion on specific GBs.

\section{EXPERIMENTAL}

Si thin films with thickness of several $\mu \mathrm{m}$ were deposited by hot-wire chemical vapor deposition (HWCVD) at NREL from pure $\mathrm{SiH}_{4}$ gas onto $\sim 100-\mathrm{nm}$ thick silicon seed layers formed by aluminum-induced crystallization (AIC) on glass substrates. The seed layers were prepared at Helmholtz Centre Berlin for Materials and in Germany [26]. The deposition process and epitaxial alignment of the HWCVD-grown layer can be found in the literature $[27,28]$. The individual grains are typically $\sim 10 \mu \mathrm{m}$ in size.

The SCM technique $[29,30]$ has been extensively used in two-dimensional characterization of carrier concentration in semiconductor devices. SCM is based on the contact mode of atomic force microscopy (AFM). Its spatial resolution is on the nanometer scale or about equal to the size of AFM tip apex. It measures the carrier concentration in a sample by measuring the highfrequency capacitance of a metal-oxide-semiconductor (MOS) structure consisting of the AFM tip, an oxide-layer on the Si film, and the Si film. Detailed description of the technique can be found in the literature $[29,30]$. The AC voltage used to drive the capacitance change was $0.5-$ $1.0 \mathrm{~V}$ in amplitude and $40-90 \mathrm{KHz}$ in frequency. The DC voltage used to drive the MOS structure to a flat band condition was -0.5 to $-1 \mathrm{~V}$ biased to the sample. A uniform and less-defective oxide layer on top of the film was critical to achieving a high-quality SCM measurement. The conventional way to get a high-quality oxide layer is to polish the film surface chemical- 
(a) AFM

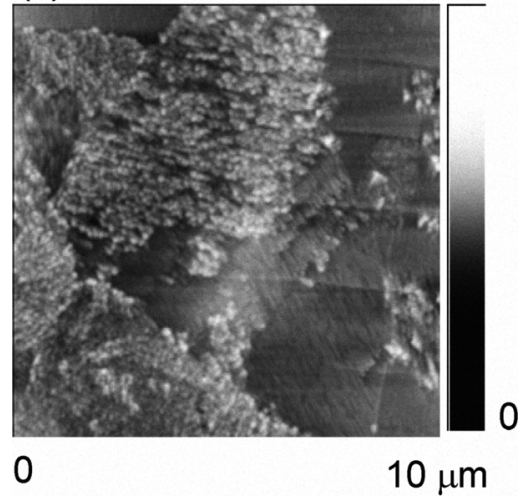

(b) AFM

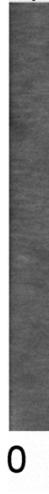

$10 \mathrm{~nm}$

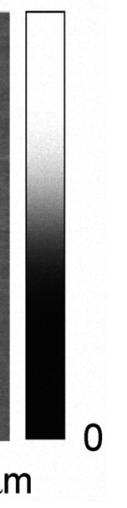

(c) SCM

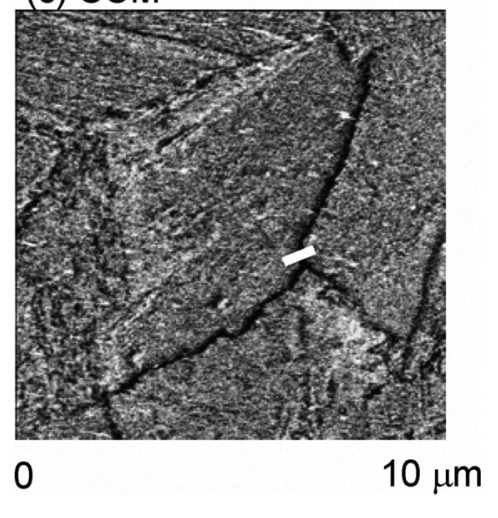

(d)

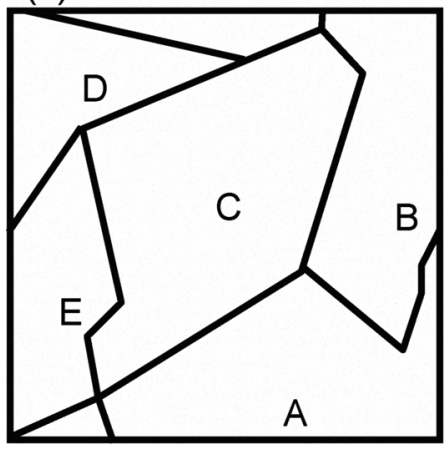

(e)

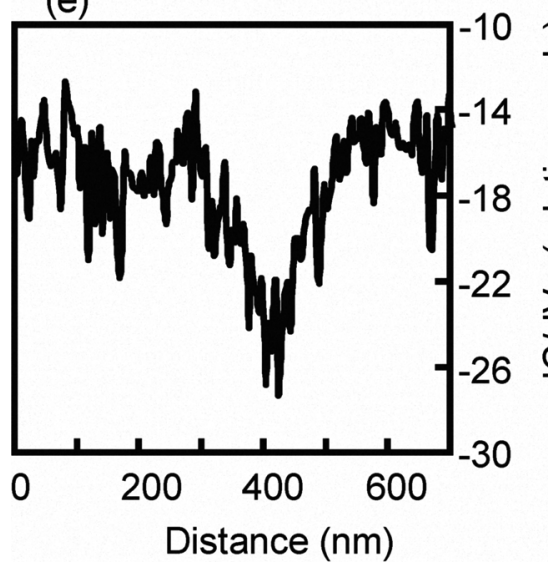

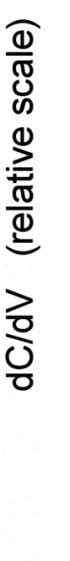

Fig. 1(a) An AFM image taken on an as-grown polycrystalline Si thin film. (b) and (c) are a pair of AFM and the corresponding SCM images taken on a polished Si film. (d) shows a map of the grain structure as identified from the carrier depletion on the GBs and from the textures shown in Fig. 1(c). (e) shows a line profile of the SCM dC/dV signal along the white short line in Fig. 1(c).

mechanically by using colloidal silica with fine $\sim 50-\mathrm{nm}$ particles. After polishing, ultraviolet irradiation treatment while heating the sample at $\sim 300^{\circ} \mathrm{C}$ was found to improve the quality of the oxide layer, as reported in Ref. 30.

EBSD [31] has been extensively used in characterizing crystal structures and grain orientations of polycrystalline films. It differs from X-ray diffraction, which measures grain orientation in a large section of sample containing a large number of microscopic grains. EBSD measures the grain orientation on individual grains with a submicrometer resolution. Using an electron gun of a scanning electron microscope (SEM), EBSD scans an electron beam on a sample, and collects Kikuchi patterns from every pixel. Subsequently, a map of the grain orientation is obtained by analyzing the Kikuchi patterns. Detailed description of EBSD can be found elsewhere [31]. A common requirement for high-quality EBSD measurement is a flat sample surface to avoid shadowing the electron diffraction beams from a rough surface. The polished Si films we used for the SCM measurement provided good sample preparation for a high-quality EBSD measurement.

\section{RESULTS AND DISCUSSION}

\subsection{Carrier Depletion by SCM Measurement}

The AFM and SCM measurements were carried out on as-grown $\mathrm{Si}$ films, and the AFM image is shown in Fig. 1(a). The image reveals that the surface morphology varies from grain to grain, depending on the grain orientation. The images show several types of surface morphologies, which may correspond to the low-index orientations in the film growth direction. However, the SCM measurement (the image is not shown) does not show clear features, due to the low-quality oxide layer on the film surface. 


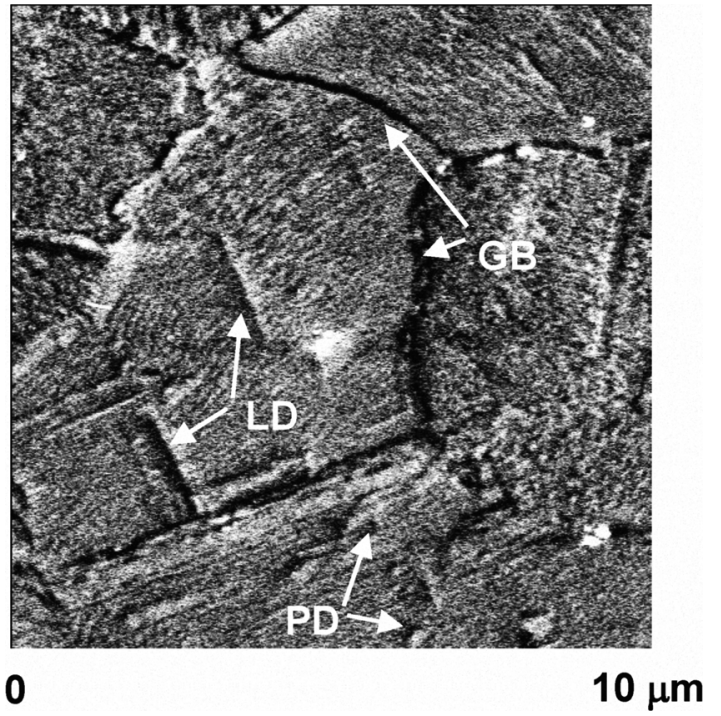

Fig. 2. An SCM image taken on a polished Si film, showing intragrain line defects (LD) and point defects (PD), as well as the surface topography-like texture on the grains.

Chemical-mechanically polishing the film surface greatly improved the quality of the SCM images. An AFM image and the corresponding SCM image on a polished sample are shown in Figs. 1(b) and 1(c). From the AFM image, it is evident that the corrugation on the film surface was reduced from $\sim 50 \mathrm{~nm}$ before polishing to $<2$ $\mathrm{nm}$ after the polishing. In the SCM image, carrier depletion on the GBs is clearly observed as dark lines. Because the film surface is flat, the GBs cannot be identified from the AFM image. However, the GBs can be recognized from the high-quality $S C M$ image. They can be identified from the depletion on the GBs or from the subtle topography-like features on the grains. We will explain the topography-like features later. An identification of the grain structure is shown in Fig. 1(d), based on the data from Fig. 1(c).

The SCM image also shows that the carrier depletion behavior is not uniform among the GBs. For example, the $G B s$ between grains $A / C, B / C$, and $A / B$ in Figs. 1(c) and 1(d) exhibit clear carrier depletion. However, the GBs between grains C/D and C/E do not exhibit significant depletion. This nonuniformity in the GB depletion demonstrates that the electronic properties of the GBs vary, possibly due to specific GB structure. A line profile along the short white line in Fig. 1(c) is shown in Fig. 1(e). It indicates that the depletion width at each side of the GB is $\sim 100 \mathrm{~nm}$.

The carrier depletion around the GB indicates an upward band bending in the $n$-type film and a negatively charged GB. The charged GB originates from deep levels in the bandgap or impurity ions acting like an acceptor. The charged deep levels are harmful for the photovoltaic performance, because they would negatively impact all the performance parameters of short-circuit current density $\left(\mathrm{J}_{\mathrm{sc}}\right)$, open-circuit voltage $\left(\mathrm{V}_{\mathrm{oc}}\right)$, and fill factor $(F F)$
[1,5-8]. It degrades $J_{s c}$ through recombination of photoexcited carriers, $V_{o c}$ through enhancing dark saturated current by the GB recombination of carrier transport, and FF through both $\mathrm{V}_{o c}$ and series resistance degradations $[1,5-8]$. On the other hand, if the GB does not show carrier depletion, the GB is not electrically active, i.e., there is no charged deep levels or impurity ions, and no electrostatic potential fluctuations on the GB. The inactive GBs should not have significant negative impact on the device performance. We believe that the carrier depletion behavior as measured by SCM can be a crucial characteristic that evaluates whether or not a GB is electrically active.

The SCM images also exhibit intragrain defects and texture-like features on the grains. An example of the SCM images is shown in Fig. 2. Four examples of the intragrain defects are indicated on the image: two line defects labeled as LD and two point defects as PD. We note that the intragrain defects are not observed on every SCM image, because only the defects at the interface of oxide-layer/Si-film or in the region close to the interface with a distance less than the depletion width can be detected by SCM. If the defects are deeper in the film's bulk than the depletion width, they are screened by carriers. SCM measures the carrier depletion on the charged defects. However, the nature of the defects is not clear, which can be vacancy, interstitial, substitutional, or agglomeration of the point defects. Similar to the GBs, these charged intragrain defects can be active recombination centers for minority carriers and can result in potential fluctuations. Therefore, the intragrain defects are considered harmful to photovoltaic performance.

The texture-like features on the grains (Fig. 2) originate from nonuniformity of the oxide layer on top of the film. These features are similar to the topographic features on the film surface [Fig. 1(a)] before the surface was polished. During the polishing, the oxide layer may have been grown nonuniformly, following the facet features of the grain orientations. This nonuniformity of the oxide layer includes layer thickness and charged defects at the interface between the oxide layer and the film, which are sensitive to the SCM measurements.

\subsection{Grain Orientations by EBSD Measurement}

With an electron beam scanning from one grain to another, the Kikuchi pattern changes its diffraction bands due to the grain orientations. The $\mathrm{GB}$ and the grain orientations adjacent to the GB can be identified by indexing the diffraction bands. A grain structure taken on a polished film is shown in Fig. 3 (a), where the GBs were identified from neighboring grains with grain misorientation angles larger than $5^{\circ}$. From the grain structure [Fig. 3(a)], one sees that the grain size is $\sim 10$ $\mu \mathrm{m}$, consistent with the AFM images [Fig. 1(a)].

We use the color scheme of inverse pole figure (IPF) to present the grain orientations in the film growth direction [Fig. 3(b)], where pure red, blue, and green colors represent the [001], [111], and [101] orientations [Fig. 3(c)]. The color of the orientation map is reddish with some blue grains and a few green ones. This 
(a)

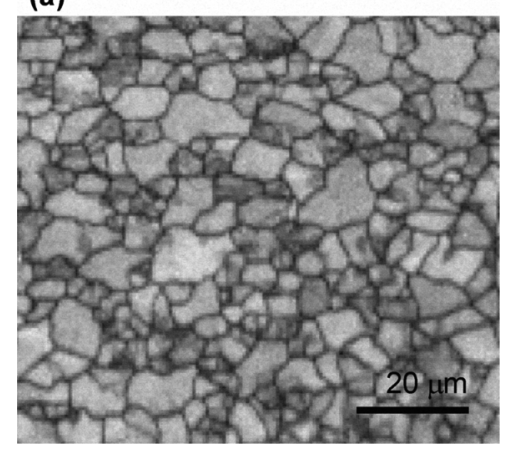

(b)

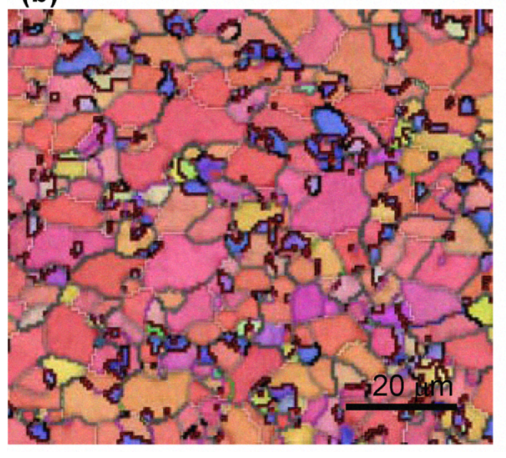

(c)

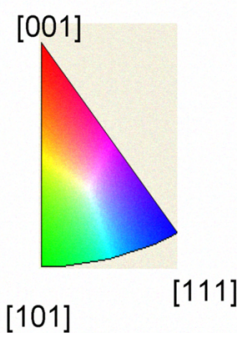

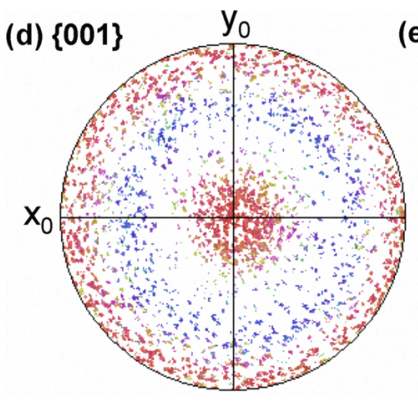

(e) $\{111\}$
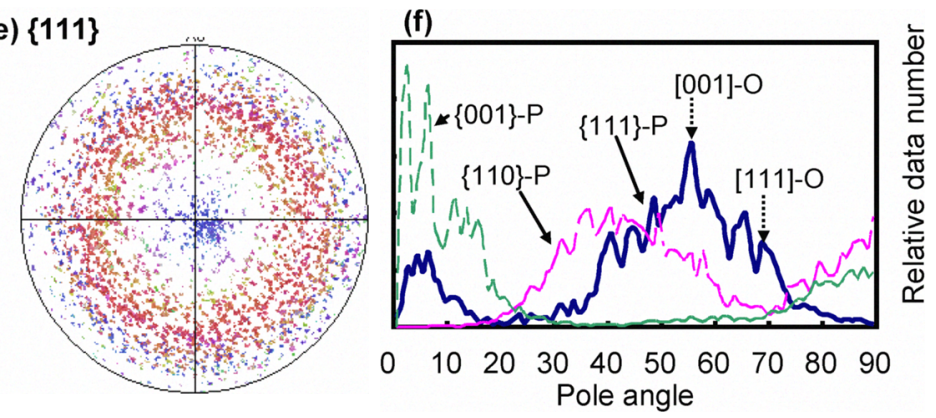

Fig. 3(a) A grain structure of the Si film measured by EBSD. (b) is the same grain structure as Fig. 3(a) with grain orientations represented by the IPF color scheme as shown in Fig. 3(c). (d) and (e) are $\{001\}$ and $\{111\}$ pole figures of the EBSD grain orientation map. (f) shows the pole figure profiles of data amount versus pole angle.

indicates that the grains are weakly [001]-oriented with misorientation angles to the [001] direction. To further present the grain orientations, pole figures in the [001] and [111] directions are shown in Fig. 3(d) and 3(e). The data points in the pole figures are rather scattered, indicating the randomness of the grain orientations. However, one sees from the $\{001\}$ pole figures that the data points are weakly concentrated in the regions of the circle center and circle edge, which correspond to the [001]-orientated grains. Also, some data points are even less concentrated around a ring inside the pole figure circle, which corresponds to the [111]-oriented grains. In the $\{111\}$ pole figure, the data points are weakly concentrated on a wide ring inside the circle that includes both [001]- and [111]-oriented grains. The profiles of data amount versus pole angle are shown in Fig. 3(f). On the profile of $\{001\}$ pole figure, most data points concentrate on regions around $0^{\circ}$ and $90^{\circ}$, which represent [001]oriented grains. However, the misorientation angle to both $0^{\circ}$ and $90^{\circ}$ is in a wide range, about $25^{\circ}$. On the profile of the $\{111\}$ figure, the data points are most concentrated around $55^{\circ}$, corresponding to the [001]oriented grains, and less concentrated around $0^{\circ}$ and $70^{\circ}$, corresponding to the [111]-oriented ones.

\subsection{Comparison of Carrier Depletion and Grain Misorientations on Individual GBs}

Figure 4 shows two pairs of SCM and EBSD images. From the grain structure in both the SCM and EBSD images, one sees that Figs. 4(a) and 4(b) were taken on the same area, and Figs. 4(c) and 4(d) on the same area. In addition to the color scheme representing the grain orientations, thin and bright lines in the EBSD maps [Figs. 4(b) and 4(d)] represent GBs with small misorientation angles, and thick and dark lines with large angles. The misorientation directions are labeled on each of the GBs. Also, the colored lines represent twin GBs, red lines the $\Sigma 3 \mathrm{GBs}$ and pink lines the $\Sigma 9 \mathrm{GBs}$. For random GBs, the GB structure is determined not only by the grain misorientation, but also by the grain facets adjacent to the GBs. However, for the twin GBs like $\Sigma 3$ and $\Sigma 9$, because the symmetry and the small formation energy at these GBs are obvious, the GB structure can be determined only from the grain misorientation.

Comparing the SCM image and the EBSD map, one sees: (1) the $\Sigma 3$ boundaries do not exhibit carrier depletion. There are many $\Sigma 3$ boundaries because of the small formation energy. However, the $\Sigma 3$ boundaries cannot be seen in the SCM images. (2) Some of the $\Sigma 9$ GBs exhibit carrier depletion, and some do not. For example, carrier depletion shows on boundaries $A$ and $C$ in Fig. 4(d), but does not show on boundaries $B$ and $D$. (3) For random GBs, we did not find a rule or a trend to describe the relationship between the carrier depletion behaviors and the grain misorientation. We compared several pairs of the SCM and EBSD images, examined in particular the grain misorientation angle and indexes with the degrees of carrier depletions, and did not find significant relationships so far. Interestingly, we found that the GBs can behave differently even for GBs that are 
(a) SCM

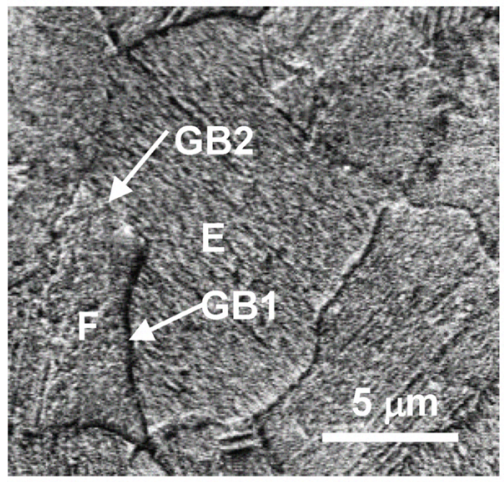

(c) SCM

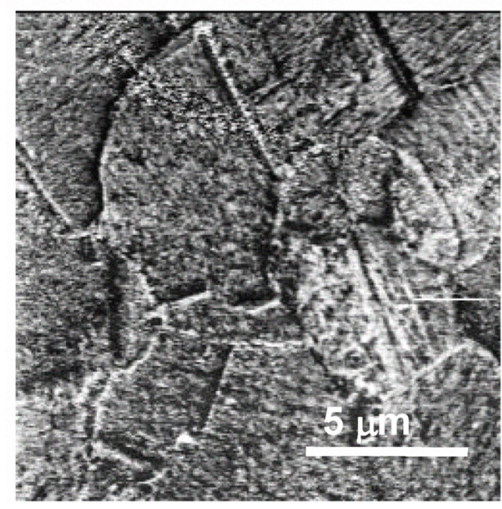

(b) EBSD

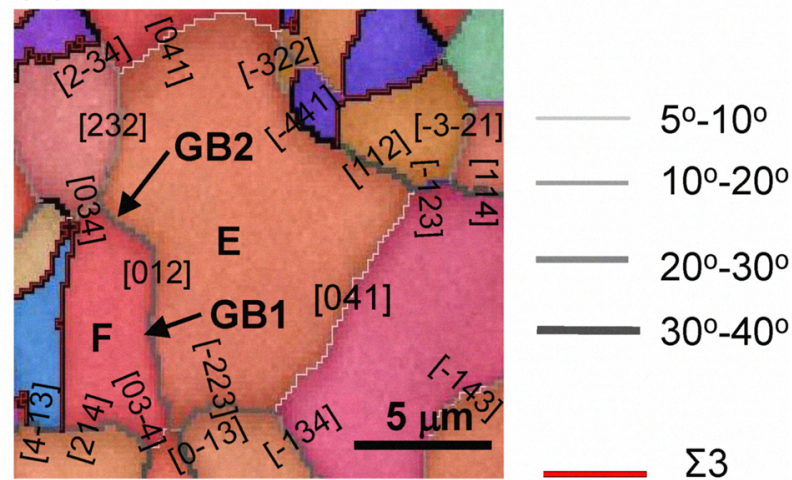

(d) EBSD

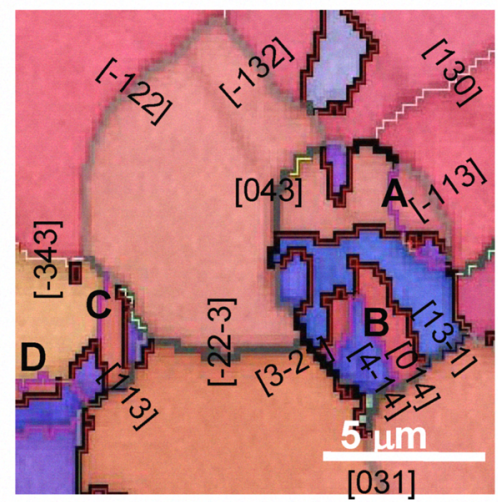

$\Sigma 9$

Fig. 4. Two pairs of SCM (a) and (c) and EBSD (b) and (d) images taken on the same area (a) and (b) and another area (c) and (d). Various lines shown in the right side of the figure represent the various GBs in Figs. 4(b) and 4(d).

between the same two grains. For example, GB1 between the lower parts of grains $E$ and $F$ in Fig. 4(b) shows clear carrier depletion. However, GB2 between their higher parts does not show significant carrier depletion. This observation clearly demonstrated that the grain misorientation by itself cannot determine the GB structure and subsequently the microelectronic properties.

Our SCM measurements show no significant carrier depletion on the $\Sigma 3$ GBs. The $\Sigma 3$ GBs are considered electrically inactive and intentionally introduced in multicrystalline Si solar cell ingots $[32,33]$. $\Sigma 3\{111\} \mathrm{GB}$ is a coherent first-order twin-boundary, the atomic structure of which contains no dangling and strained bonds [11]. This atomic structure is believed to have a very minor effect on the electronic structure, and it does not create charged deep levels. Also, because there are no dangling bonds and strained lattice spacing, the energy is high to accommodate impurity ions in the $\mathrm{GB}$. Therefore, there is no significant carrier depletion on $\Sigma 3\{111\}$ GBs. On the other hand, the structure model of $\Sigma 3\{112\}$ GB suggests that the GB contains strained bonds but no dangling bonds [13]. Chen et al. probed weak, if any, recombination at both the $\Sigma 3\{111\}$ and $\Sigma 3\{112\}$ GBs in clean multicrystalline Si samples [14]. However, the carrier recombination was much enhanced in contaminated samples, and the recombination strength is much stronger on the $\Sigma 3\{112\}$ than on the $\Sigma 3\{111\}$ GBs. This corresponds to the stronger impurity gettering property of the $\Sigma 3\{112\}$ GB [14], due to the strained lattice spacing [13].

Two alternative possible GB structures exist for the $\Sigma 9\{221\}$ GBs; both contain strained bonds, but no dangling bonds $[11,12]$. The incoherent structure of the GB is essentially similar to $\Sigma 3\{112\}$, containing five- and seven-membered rings at the core of the structure unit $[11,12]$. Our SCM measurement shows that some of the $\Sigma 9\{221\}$ GBs are charged and some are not, suggesting that the intrinsic $\Sigma 9\{221\}$ GB does not create charged deep levels, which is supported by the GB structures containing no dangling bonds. Impurity gettering can be responsible for the measured carrier depletion as the strained lattice spacing could enhance the impurity gettering at the $G B$, and result in increased carrier depletion. Our SCM measurements also indicate a stronger gettering strength on the $\Sigma 9\{221\}$ GBs than on either the $\Sigma 3\{111\}$ or $\Sigma 3\{112\}$ GBs, which is consistent with the literature [14].

For random GBs, the structure is rather complicated; it depends not only on the grain misorientation, but also on the GB planes. The electronic properties can be even more complicated, due to their 
strong impurity gettering. For specific GBs, the structural and electronic properties depend on both misorientations and $\mathrm{GB}$ planes. For a large number of $\mathrm{GBs}$, the $\mathrm{GB}$ properties may also have a statistical relationship with misorientations, if considering that a GB with a large misorientation angle about a high-index direction has, in general, high densities of dangling and strained bonds. A large number of comparisons between the SCM and EBSD measurements and a proper quantitative measurement of the carrier depletion are necessary for this study.

\section{SUMMARY}

Carrier depletion behavior of individual GBs of polycrystalline Si films were measured and resolved by using SCM. The measurement revealed that the GBs exhibit carrier depletion due to charged deep levels or impurity ions, and that the depletion is highly nonuniform among the GBs due to the varieties of GB structures. The structure of the films was investigated by using EBSD measurement. It showed that the films are weakly [001]oriented with small fractions of the grains in [111] and [110] orientations. Comparison of the SCM and EBSD measurements taken on the same film areas revealed: (1) the $\Sigma 3$ GBs do not exhibit carrier depletion, and thus are electrically inactive. (2) Some $\Sigma 9$ GBs exhibit carrier depletion and some do not, suggesting that the intrinsic $\Sigma 9 \mathrm{GBs}$ are electrically inactive and the carrier depletion observed on some of these is due to impurity gettering at the GBs. (3) No significant relationship between the carrier depletion behavior and the grain misorientation was found so far for random GBs. (4) The carrier depletion does not depend only on the grain misorientaion, but also, on the facet where the GBs are taken.

\section{ACKNOWLEDGEMENT}

The authors thank C.W. Teplin at NREL and Stefan Gall at Helmholtz Centre Berlin for Materials and Energy in Germany for providing the silicon films. This work was supported by the U.S. Department of Energy under Contract No. DOE-AC36-08GO28308 with NREL.

\section{REFERENCES}

[1] T. Yamazaki, Y. Uraoka, and T. Fuyuki, Thin Solid Films 487, 26 (2005).

[2] N. Kawamoto, A. Matsuda, N. Matsuo, Y. Seri, T. Nishimori, Y. Kitamon, H. Matsumura, H. Hamada, and T. Miyoshi, Jpn. J. Appl. Phys. 45, 2726 (2006).

[3] A.G. Aberle, Proc. $4^{\text {th }}$ World Conf. Photovoltaic Energy Conversion, Hawaii, 2006, p.1481.

[4] P.A. Basore, Proc. $19^{\text {th }}$ European Photovoltaic Solar Energy Conf., Paris, 2004, p.455.

[5] K. Kurobe, Y. Ishikawa, Y. Yamamoto, T. Fuyuki, and H. Matsunami, Solar Energy Material \& Solar Cells 65, 201 (2001).

[6] T. Matsui, T. Yamazaki, A. Nagatani, K. Kino, H. Takakura, and Y. Hamakawa, Solar Energy Materials \& Solar Cells 65, 87 (2001).
[7] Y. Ishikawa, Y. Yamamoto, T. Hatayama, Y. Uraoka, and T. Fuyuki, Jpn. J. Appl. Phys. 40, 6783 (2001).

[8] G. Beaucarne, S. Bourdais, A. Slaoui, and J. Poortmans, Appl. Phy. A79, 469 (2004).

[9] G. Beaucarne, S. Bourdais, A. Slaoui, and J. Poortmans, Proc. $28^{\text {th }}$ IEEE PVSC, Alaska, 2000, p.128.

[10 E. Christoffel, M. Rusu, A. Zerga, S. Bourdais, S. Noël, and A. Slaoui, Thin Solid Films 403-403, 258 (2002).

[11] For a review see: D.B. Holt and B.G. Yacobi, Extended Defects in Semiconductors, (Cambridge University Press, Cambridge, UK, 2007), p.292-331.

[12] For a review see: C. Grovenor, J. Phys. C18, 4079 (1985).

[13] H. Sawada and H. Ichinose, Scripta Mater. 44, 2327 (2001).

[14] J. Chen, T. Sekiguchi, D. Yang, F. Yin, K. Kido, and S. Tsurekawa, J. Appl. Phys. 96, 5490 (2004).

[15] A. Bay and G. Nouet, J. Phys. 63, 435 (1988).

[16] Z.-J. Wang, A. Tsurekawa, K. Ikeda, T. Sekigichi, and T. Watanabe, Interface Science 7, 197 (1999).

[17] D. S. Kyriakos, A. N. Anagnostopoulos, Ph. Komninous, and B. Ploss, Semicond. Sci. Technol. 6, 607 (1991).

[18] J.Y.W. Seto, J. Appl. Phys. 46, 5247 (1975).

[19] B. Warren, N.M. Jackson, and D.K. Biegelsen, Appl. Phys. Lett. 43, 195 (1983).

[20] J. Werner and M. Peisl, Phys. Rev. B31, 6881 (1985).

[21] Y. Alpern and J. Shappir, J. Appl. Phys. 63, 2694 (1988)

[22] H. Hasegawa, M. Arai, and Y. Kurata, J. Appl. Phys. 71, 1462 (1992).

[23] F. Cleri, P. Keblinski, L. Colombo, S.R. Phillpot, and D. Wolf, Phys. Rev. B57, 6247 (1998).

[24] S. Ostapenko, Applied Physics A69, 225 (1999).

[25] W. Choi, V. Matias, J.-K. Lee, and A.T. Findikoglu, Appl. Phys. Lett. 87, 152104 (2005).

[26] S. Gall, J. Schneider, J. Klein, K. Hübener, M. Muske, B. Rau, E. Conrad, I. Sieber, K. Petter, K. Lips, M. Stöger-Pollach, P. Schattschneider, and W. Fuhs, Thin Solid Film 511-512, 7 (2006).

[27] Q. Wang, C.W. Teplin, P. Stradins, B. To, K.M. Jones, and H.M. Branz, J. Appl. Phys. 100, 093520 (2006).

[28] C.W. Teplin, H.M. Branz, K.M. Jones, M.J. Romero, P. Stradins, and S. Gall, Mat. Res. Soc. Symp. Proc. 989, 133 (2006).

[29] C.C. Williams, W.P. Hough, and S.A. Rishton, Appl. Phys. Lett. 55, 203 (1989).

[30] V.V. Zavyalov, J.S. McMurray, and C.C. Williams, Rev. Sci. Instruments 70, 158 (1999).

[31] A.J. Schwartz, M. Kumar, and B.L. Adams, Electron Backscatter Diffraction in Material Science, (Kluwer Academic/Plenum Publishers, New York, 2000).

[32] A.L. Endrös, Solar Energy Material \& Solar Cells 72, 109 (2002).

[33] M. Kitamura, N. Usami, T. Sugawara, K. Kutsukake, K. Fujiwara, Y. Nose, T. Shishido, and K. Nakajima, J. Crystal Growth 280, 419 (2005). 